\title{
Evaluation of Diagnostic Accuracy and Radiation Exposure of Dual-Energy Computed Tomography (DECT) in the Course of Chronic Thromboembolic Pulmonary Hypertension (CTEPH) Evaluation der diagnostischen Genauigkeit und der Strahlendosis der Dual-Energy-Computertomografie (DECT) bei chronisch thromboembolischer pulmonaler Hypertonie (CTEPH)
}

Authors

Armin Schüßler ${ }^{1,2}$, Manuel Richter 2, 3, Khodr Tello², 3, Dagmar Steiner 2, 4, Werner Seeger 2, 3, Gabriele Anja Krombach, 2 , Fritz Christian Roller ${ }^{1,2}$

Affiliations

1 Department of Diagnostic and Interventional Radiology, University Hospital Gießen, Justus Liebig University, Gießen, Germany

2 Member of the German Centre for Lung Research (DZL), Germany

3 Department of Internal Medicine II, University Hospital Gießen, Justus Liebig University, Gießen, Germany

4 Department of Nuclear Medicine, University Hospital Gießen, Justus Liebig University, Gießen, Germany

Key words

DECT, CTEPH, PBV, iodmap, pulmonary hypertension

received 06.11.2020

accepted 12.04.2021

published online 17.06.2021

Bibliography

Fortschr Röntgenstr 2021; 193: 1318-1326

DOI 10.1055/a-1502-7541

ISSN 1438-9029

(c) 2021. Thieme. All rights reserved.

Georg Thieme Verlag KG, Rüdigerstraße 14,

70469 Stuttgart, Germany

Correspondence

Armin Schüßler

Department of Diagnostic and Interventional Radiology

University Hospital Gießen

Justus Liebig University, Klinikstr. 33, 35385 Gießen, Germany Tel.: +49/641/985/41818

armin.schuessler@radiol.med.uni-giessen.de
ABSTRACT

Purpose The purpose of this study was to assess the diagnostic accuracy of computed tomography pulmonary angiogram (CTPA) including dual energy and reconstruction of iodine maps for diagnosing CTEPH. This method for detecting embolisms and perfusion failures was compared with V/Q-SPECT. An additional purpose was to compare the applied radiation dose of both techniques.

Materials and Methods 71 patients (49 women) with suspected CTEPH were included in this prospective study. The patients received a V/Q-SPECT and a dual-energy CTPA. lodine maps were reconstructed from the data set. CTPA and the iodine maps were read by an experienced radiologist unaware of the clinical information as well as the results of the V/QSPECT. Results were compared to the V/Q-SPECT. DLP and the applied amount of radionuclides (MAA, Technegas) were obtained for comparison of radiation dose.

Results For the diagnosis of CTEPH, the sensitivity of DECT was 1.000 , specificity 0.966 , PPV 0.867 and NPV 1.000, respectively. There was not a considerable difference in the $\mathrm{x}$-ray exposure between the DECT examination and the V/QSPECT (1.892 mSv vs. $1.911 \mathrm{mSv} ; \mathrm{p}=0.6115)$. Both examination modalities were highly consistent regarding the classification of pathological segments ( $1177 / 1278$ segments, $92,09 \%, \mathrm{k}=0,5938$ ).

Conclusion This study presents the DECT, in combination with reconstructed iodine maps, as a potential alternative to the current imaging technique of first choice, V/Q-SPECT. For creating future prospective diagnostic algorithms, the implementation of DECT screening with iodine maps should be considered.

Key Points:

- DECT correctly identified all CTEPH patients.

- There is substantial agreement between DECT and $\mathrm{V} / \mathrm{Q}-\mathrm{SPECT}$ in the classification of pathological segments.

- There is no significant difference in radiation exposure during DECT examination and V/Q-SPECT examination.

- Reduced radiation dose does not negatively impact image quality. 


\section{Citation Format}

- Schüßler A, Richter M, Tello K et al. Evaluation der diagnostischen Genauigkeit und der Strahlendosis der DualEnergy-Computertomografie (DECT) bei chronisch thromboembolischer pulmonaler Hypertonie (CTEPH). Fortschr Röntgenstr 2021; 193: 1318-1326

\section{ZUSAMMENFASSUNG}

Ziel Ziel der Studie war es herauszufinden, wie exakt die diagnostische Genauigkeit der computertomografischen Pulmonalisangiografie (CTPA) unter Hinzunahme von Dual-Energy und Rekonstruktionen von Jodkarten bei der Diagnosestellung einer CTEPH ist. Zudem wurde die Übereinstimmung der CTPA mit der Ventilations-/Perfusionsszintigrafie (V/Q-SPECT) bei der Detektion von Embolien und Perfusionsausfällen untersucht. Ein weiteres Ziel war es, die applizierte Strahlenexposition beider Techniken miteinander zu vergleichen.

Material und Methoden 71 Patienten (49 Frauen) mit dem Verdacht auf eine CTEPH wurden in diese prospektive Studie eingeschlossen. Die Patienten erhielten eine V/Q-SPECT und eine Dual-Energy-CTPA und hieraus rekonstruierte Jodkarten.
Die Analyse der CTPA und der Jodkarten erfolgte durch einen erfahrenen Radiologen, verblindet zu den klinischen Angaben und in Unkenntnis des V/Q-SPECT-Befundes. Die Ergebnisse wurden mit dem Befund der V/Q-SPECT verglichen. DLP und die applizierten Radionuklidmengen (MAA, Technegas) wurden zur Ermittlung der Strahlenexposition erfasst.

Ergebnisse Die DECT erzielte eine Sensitivität von 1,000, eine Spezifität von 0,966, einen PPV von 0,867 und einen NPV von 1,000. Zwischen den beiden Untersuchungsmodalitäten gab es, in Bezug auf die Strahlenexposition, nahezu keinen Unterschied (1,911 mSv vs. 1,892 mSv). Die Untersuchungsmodalitäten zeigten im Hinblick auf Perfusionsdefizite eine hohe Übereinstimmung (1177/1278 Segmente, 92,09\%; $\mathrm{K}=0,5938$ ).

Schlussfolgerung Die DECT präsentierte sich, in Kombination mit den Jodkarten, als potenzielle Alternative zum gegenwärtigen bildgebenden Verfahren der ersten Wahl, der V/Q-SPECT. Bei der Erstellung zukünftiger diagnostischer CTEPH-Untersuchungsalgorithmen sollte die Implementierung der DECT erwogen werden.

\section{Introduction}

Chronic thromboembolic pulmonary hypertension (CTEPH) is often first diagnosed at a very late stage. This disease is defined as an increase in the mean pulmonary-arterial pressure (mPAP) to more than $25 \mathrm{mmHg}$ with persistent perfusion deficits or vascular lesions after three months of anticoagulation therapy $[1,2]$. The treatment of first choice is pulmonary endarterectomy (PEA). If the patient is not suitable for this procedure, pulmonary balloon angioplasty (BPA) should be considered. If this treatment option is also not possible due to the location of the occlusions or other factors, treatment with medication is the only option [3]. Untreated, CTEPH has a poor prognosis and patients can die within several years [4]. Those affected report only progressive exertional dyspnea. This nonspecific symptom occurs in almost all forms of pulmonary hypertension and complicates differentiation from other cardiovascular diseases with similar symptoms $[3,5]$. At present, ventilation/perfusion scintigraphy (as V/QSPECT where possible) is recommended for the screening of patients with pulmonary hypertension and indication of CTEPH [3]. If perfusion defects with preserved ventilation are confirmed, the referral to a lung center for further diagnostic workup via CT pulmonary angiography (CTPA) or invasive visualization of the pulmonary vessels (DSA) as well as right-heart catheter examination is recommended $[6,7]$.

Using the dual-energy technique and the corresponding software, it is possible to detect iodine distribution in the lung after administration of a contrast agent containing iodine and to make a conclusion about the perfusion within the lung parenchyma [8]. This additional information can be decisive with respect to CTEPH. Newer studies show that the reconstruction of iodine maps improves the diagnosis of CTPA, particularly when examining indi- vidual lung segments and peripheral lung perfusion [9]. In 2014, the working group of Dournes et al. was able to show moderate agreement between V/Q-SPECT and the iodine maps generated from contrast-enhanced DECT [10].

The goal of this study was therefore to determine how exact DECT (CTPA and iodine map) is for diagnosing CTEPH and to examine the comparability of the two examination modalities. The agreement between DECT and V/Q-SPECT regarding the detection of embolisms and perfusion deficits was determined for this purpose. Further goals were to compare the radiation exposure of the two techniques with one another and to determine the image quality of DECT and iodine maps.

\section{Materials and Methods}

The study was approved by the ethics committee and lasted for a period of two years. All patients with suspicion of CTEPH scheduled to undergo a DECT examination with contrast agent and a V/Q-SPECT examination were prospectively included in the study. The time between the two examinations could not exceed 14 days. Contraindications for the administration of a contrast agent containing iodine resulted in exclusion from the study. Patients were examined using a standardized examination protocol on a third-generation dual-energy computed tomography unit (SOMATOM Force, Siemens Healthineers, Erlangen, Germany) and a SPECT-/CT scanner (Symbia, Siemens Healthineers, Erlangen, Germany). In the examination using the dual-energy technique with a maximum field of view (FoV) of $355 \mathrm{~mm}$, the first X-ray tube was set to $90 \mathrm{kV}$ with a maximum reference value of $60 \mathrm{mAs}$ and the second $\mathrm{X}$-ray tube was set to $150 \mathrm{kV}$ with a tin filter and a maximum reference value of $46 \mathrm{mAs}$. The iterative reconstruction module (advanced modeled iterative reconstruction ADMIRE, 
Siemens Healthineers, Erlangen, Germany) was set to a strength of three to five. All patients were examined in a supine position with the arms positioned above the head from cranial to caudal. The examination was performed during deep inspiration breathhold. Contrast agent was administered intravenously via an indwelling cannula typically placed in the cubital vein with a size of at least $18 \mathrm{G}$ and a constant flow rate of $3.5 \mathrm{ml}$ per second. $60 \mathrm{ml}$ of a contrast agent (Ultravist-370, Bayer Vital, Leverkusen, Germany) and a $50-\mathrm{ml} \mathrm{NaCl}$ - bolus were administered. With the help of the ROI, the Hounsfield unit in the pulmonary trunk was measured during the monitoring phase in short intervals (1 frame/s) and a pulmonary arterial phase was acquired. $220 \mathrm{HU}$ in the ROI was defined as the cut-off value with a subsequent start delay of 10 seconds. CTPA included complete visualization of the scan region from the apex of the lung to the base of the lung. lodine maps were reconstructed on the coronal, sagittal, and transverse plane for every patient. The slice thickness and slice increment were $4 \mathrm{~mm}$. A postprocessing program (syngo.via/ Dual-Energy-CT, Siemens Healthineers, Erlangen, Germany) was used for the reconstruction of the iodine maps.

The V/Q-SPECT scan was also acquired with the patient in a supine position with the arms extended in a cranial direction. Ventilation scintigraphy in which the aerosol ( $T c^{99} \mathrm{~m}$-Technegas) was inhaled three times via the mouth with the nose closed was initially performed. Patients were examined using a $360^{\circ}$ technique with 60 projections and a matrix of $128 \times 128$ pixels. The maximum projection duration was set to 60 seconds or 50,000 counts in anterior projection. The energy window was set to $140 \mathrm{keV}$ with a width of $15 \%$. Perfusion scintigraphy was then performed. The radiopharmacon ( $\mathrm{Tc}^{99 \mathrm{~m}}$-MAA) was administered via an indwelling cannula typically placed in the cubital vein. The examination was also performed using a $360^{\circ}$ technique with 60 projections and a matrix of $64 \times 64$ pixels. The maximum projection duration was set to 60 seconds or 50,000 counts in anterior projection. The amount of $\mathrm{Tc}^{99 \mathrm{~m}}$-MAA had to be at least four times greater than the amount of aerosol that was inhaled. Coronal, sagittal, and transverse planes were then reconstructed for the ventilation and perfusion scintigraphy examination.

The CTPA examination and the iodine maps were analyzed by an experienced radiologist blinded to the clinical data and the V/Q-SPECT finding. Patients were classified as "suspicious for CTEPH" when perfusion deficits were present in one segment or at least two subsegments in the iodine map and/or thromboembolic material could be directly detected in the pulmonary arteries and were accompanied by a sudden change in the diameter of the peripheral pulmonary arteries and dilation of the pulmonary trunk (>30 mm). The V/Q-SPECT scan was analyzed by two nuclear medicine physicians in consensus and was evaluated as "suspicious for CTEPH" in the case of at least one segmental or two subsegmental perfusion defects. The conclusion reached by the interdisciplinary CTEPH conference after reviewing all findings (right heart catheter, V/Q-SPECT, CTPA, DSA) was considered the final diagnosis.
- Table 1 Overview of diagnoses.

\begin{tabular}{|l|c|c|}
\hline & $\mathbf{n}=\mathbf{7 1}$ & $\%$ \\
\hline CTEPH & 13 & 18.3 \\
\hline $\begin{array}{l}\text { Pulmonary hypertension of a } \\
\text { different cause }\end{array}$ & 19 & 26.8 \\
\hline Emphysema & 12 & 16.9 \\
\hline Other disease & 27 & 38.0 \\
\hline
\end{tabular}

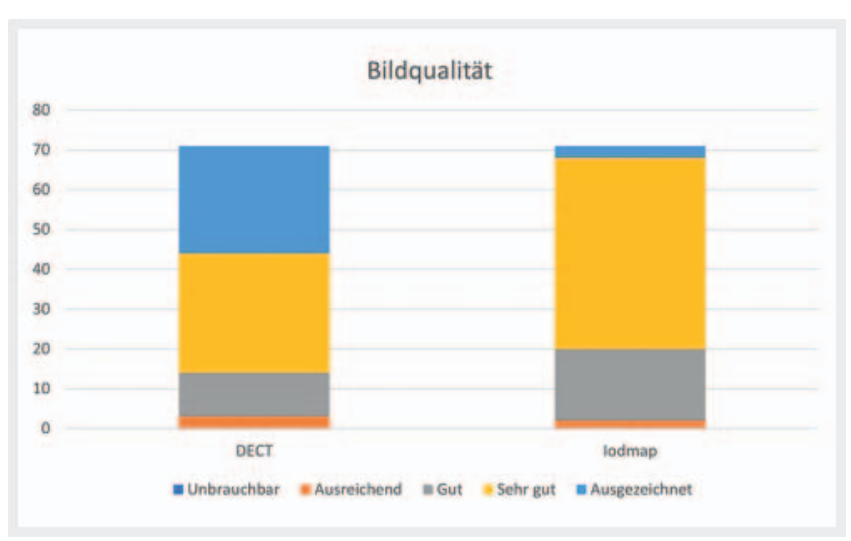

- Fig. 1 Image quality: graphical overview of DECT and iodmap.

\section{Statistical analysis}

Age, sex, and day of examination were documented. The amount of $T c^{99 m}-M A A$ and $T c^{99 m-T e c h n e g a s ~ t h a t ~ w a s ~ a d m i n i s t e r e d ~ w a s ~}$ recorded with nuclear medicine imaging and the dose-length product (DLP) was recorded with radiological imaging. To achieve a direct comparison between the modalities, the equivalence dose was calculated with the help of the amount of radionuclide that was administered and the DLP. Moreover, the radiological as well as nuclear medicine finding for every lung segment was recorded for each patient. Due to the low spatial resolution and the occurrence of cardiac artifacts on V/Q-SPECT, segments seven and eight in the right half of the lung were combined so that in total 18 lung segments were documented on radiological and nuclear medicine imaging for every patient. The diagnosis of CTEPH was made separately for each modality, radiology and nuclear medicine. The image quality of the CT examination and the reconstructed iodine map were rated using a 5-point Likert scale $(0$ points $=$ not diagnostic, $1=$ sufficient, 2 = good, 3 = very good, 4 = excellent).

The Mann-Whitney U-test was used for the biometric evaluation of radiation doses. The agreement between the two examination modalities regarding lung segments was calculated by the Cohen's kappa. Moreover, the two-sample test was used for the analysis of the patient group and the Gaussian distribution for the distribution of thromboembolic occlusions. Two statistics programs (Past3, Hammer \& Harper, Oslo, Norway and Prism8, GraphPad Software GmbH, San Diego, USA) were used for the stochastic calculation. 

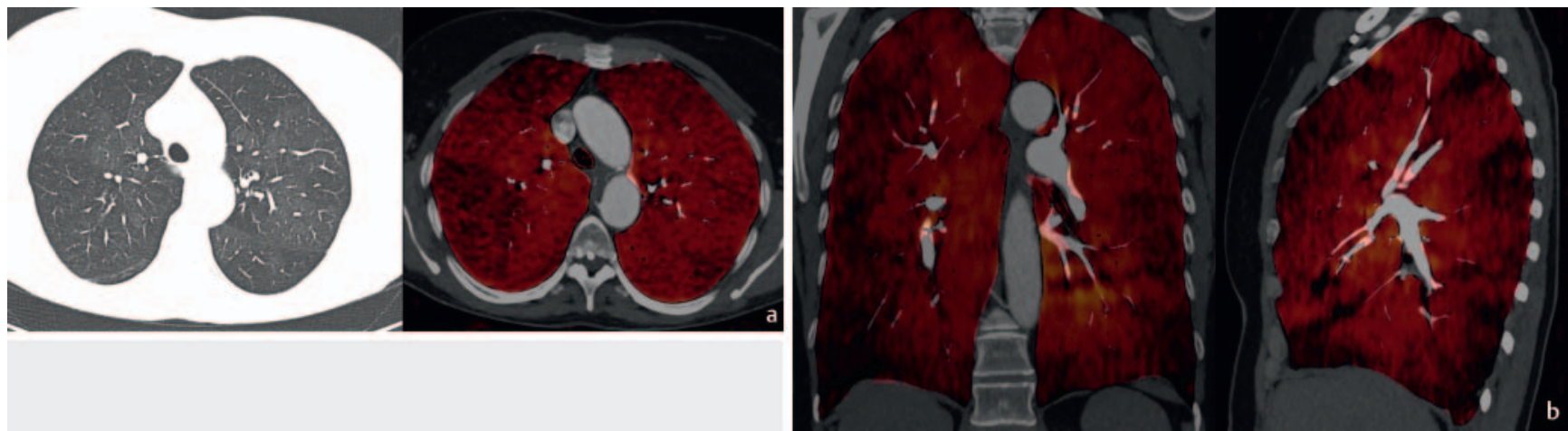

- Fig. 2 a CTEPH: Left: CT slice (lung window) at the level of the arcus aortae in a CTEPH patient. Slightly visible mosaic attenuation. Right: corresponding iodmap with multiple clearly visible wedge-shaped defects. b: CTEPH: Left: Coronal reconstruction: Almost the entire pulmonary periphery is displaying defects. Right: Sagittal reconstruction. Multiple extensive wedge-shaped lesions are clearly evident dorsal and ventral.

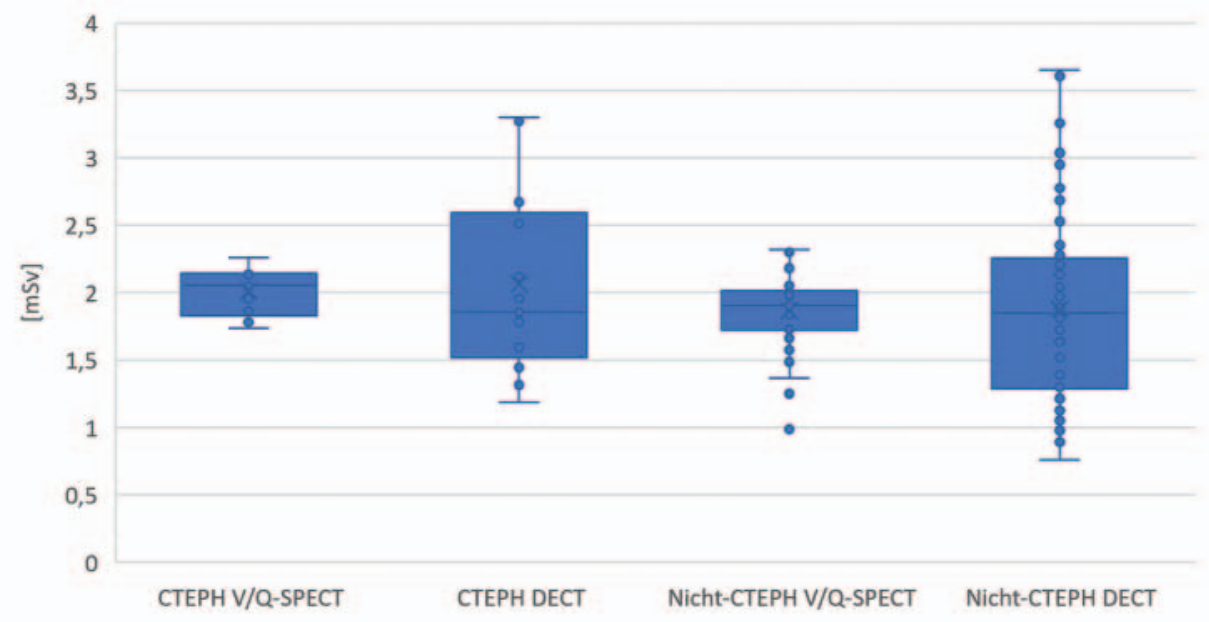

- Fig. 3 X-ray exposure: Group CTEPH and non-CTEPH.

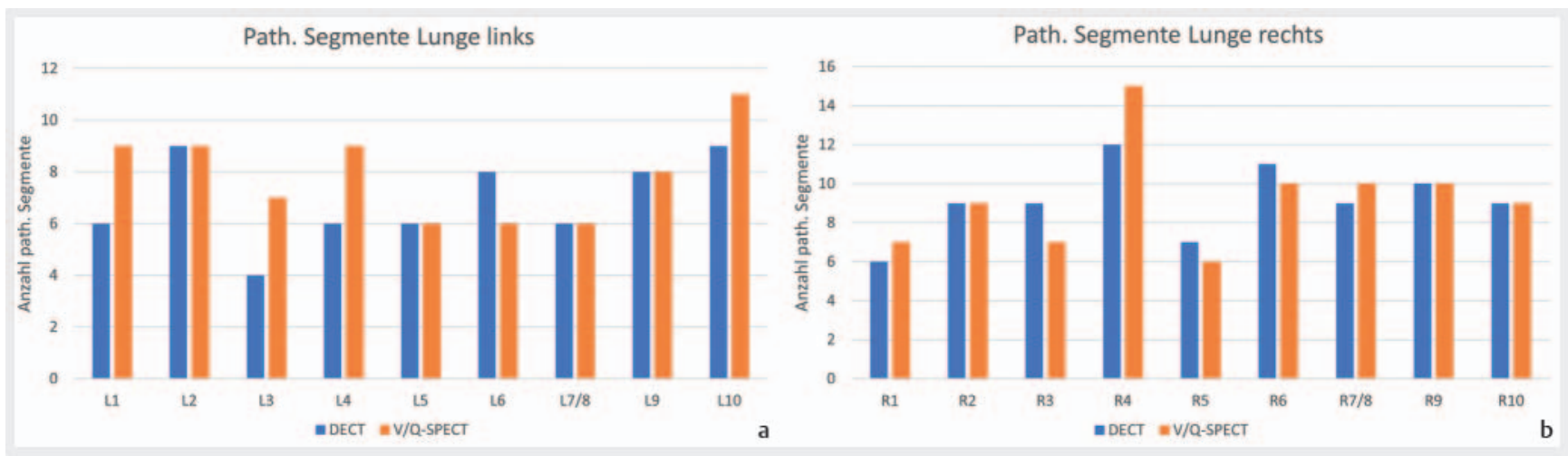

- Fig. 4 a Distribution of pathological segments of the left lung $\mathbf{b}$ : Distribution of pathological segments of the right lung.

\section{Results}

In total, 71 patients (49 females) with an average age of 63 years $+1-15.3$ standard deviation were included in the study. The youngest patient was 26 years old and the oldest was 85 years old. Two comparison groups were created: Patients without CTEPH $(n=58)$ and patients with CTEPH $(n=13)$. In the group of patients without $\mathrm{CTEPH}, 19$ had a different form of pulmonary hypertension (mPAP $>25 \mathrm{mmHg}$ in the right heart catheter 
- Table 2 Agreement between DECT and V/Q-SPECT.

\begin{tabular}{|c|c|c|c|c|}
\hline & DECT & V/Q-SPECT & agreement [\%] & к \\
\hline $\mathrm{n}$ (patients) & 71 & 71 & & \\
\hline segments & 1278 & 1278 & 92.09 & 0.5938 \\
\hline CTEPH segments & 234 & 234 & 74.79 & 0.4978 \\
\hline CTEPH lobes & 65 & 65 & 84.62 & 0.5259 \\
\hline non-CTEPH segments & 1044 & 1044 & 96.74 & -0.0189 \\
\hline segment L1 & 71 & 71 & 92.96 & 0.6324 \\
\hline segment L2 & 71 & 71 & 94.37 & 0.7482 \\
\hline segment L3 & 71 & 71 & 95.77 & 0.7091 \\
\hline segment L4 & 71 & 71 & 95.77 & 0.7799 \\
\hline segment L5 & 71 & 71 & 91.55 & 0.4574 \\
\hline segment L6 & 71 & 71 & 97.18 & 0.8438 \\
\hline segment $\mathrm{L} 7 / 8$ & 71 & 71 & 91.55 & 0.4574 \\
\hline segment L9 & 71 & 71 & 91.55 & 0.5808 \\
\hline segment L10 & 71 & 71 & 94.37 & 0.7701 \\
\hline segment R1 & 71 & 71 & 92.96 & 0.5803 \\
\hline segment R2 & 71 & 71 & 94.37 & 0.7482 \\
\hline segment R3 & 71 & 71 & 91.55 & 0.5817 \\
\hline segment R4 & 71 & 71 & 90.14 & 0.6839 \\
\hline segment R5 & 71 & 71 & 95.77 & 0.7488 \\
\hline segment R6 & 71 & 71 & 90.14 & 0.6123 \\
\hline segment R7/8 & 71 & 71 & 92.09 & 0.6993 \\
\hline segment R9 & 71 & 71 & 97.18 & 0.8851 \\
\hline segment R10 & 71 & 71 & 94.37 & 0.7482 \\
\hline
\end{tabular}

without perfusion deficits on imaging) and 12 patients had emphysema. The findings for the other 27 patients could not be assigned to any of the specified groups or did not show any pathology ( $\triangleright$ Table 1$)$.

The average age and the sex distribution within these groups did not yield any significant differences (age: $63.6+/-15.6$ vs. $60.4+/-14.1 ; p=0.49$, sex (f/m): $40 / 18$ vs. $9 / 4 ; p=0.18)$.

The image quality of DECT and the iodine map was recorded using a Likert 5-point scale. The image quality of DECT was largely rated as "very good" or "excellent". The iodine maps were largely rated as "very good" ( $>$ Fig. $\mathbf{1}$ ).

No patients had to be excluded due to a limited FOV of the second X-ray tube. This was often a problem in the case of firstgeneration DECT scanners. Representative images are shown in - Fig. 2a, b. There was almost no difference in relation to radiation exposure $(1.911+/-0.68 \mathrm{mSv}$ vs. $1.892+/-0.25 \mathrm{mSv}$; $\mathrm{p}=0.6115$ ) between the two examination modalities. Also, with respect to the two groups, $\mathrm{CTEPH}$ and non-CTEPH, there was no significant difference $(2.064+/-0.68 \mathrm{mSv}$ vs. $2.014+/-0.17 \mathrm{mSv}$, $\mathrm{p}=0.5727 ; 1.877+/-0.26 \mathrm{mSv}$ vs. $1.864+/-0.68 \mathrm{mSv}$, $\mathrm{p}=0.7135)(\triangleright$ Fig. 3$)$.

In the comparison of the two examination modalities with respect to perfusion deficits, there was high agreement between the methods. In total, 1278 lung segments showed agreement between the two modalities (1177/1278 segments, $92.09 \%$, $\mathrm{K}=0.5938) . \mathrm{V} / \mathrm{Q}$-SPECT categorized 154 segments and DECT categorized 144 segments as pathological. The agreement regarding reporting for the individual segments between the two modalities was also over $90 \%$. Segment six on the left side and segment nine on the right side showed the highest agreement $(69 / 71$ segments, $97.18 \%, \mathrm{~K}=0.8438$ and $69 / 71$ segments, $97.18 \%, \mathrm{k}=0.8851)$. The agreement for the group of $\mathrm{CTEPH}$ patients was 175 of $234(74.79 \%, \kappa=0.4978)$ for the lung segments and 55 of $65(84.62 \%, \mathrm{k}=0.5259)$ for the lung lobes ( $\triangleright$ Table 2). To determine whether there are segments or lobes that are affected particularly frequently so that conclusions about the hemodynamic conditions within the lungs can be made, the distribution of perfusion deficits was also considered. A particular accumulation was seen in segment 4 on the right side ( $\vee$ Fig. 4a, b). The lung lobe result did not show any particular predilection site.

All CTEPH patients $(n=13)$ were able to be detected with DECT (sensitivity 1.000 , positive predictive value (PPV) 0.867 ). Two patients were detected as false positives and 56 as true negatives (specificity 0.966 , negative predictive value (NPV) 1.000) $(\vee$ Table 3). Pulmonary arterial webs were seen in the two false- 
- Table 3 Sensitivity/specificity of DECT.

\begin{tabular}{|l|l|l|}
\hline & $\begin{array}{l}\text { positive } \\
\text { (DECT) }\end{array}$ & $\begin{array}{l}\text { negative } \\
\text { (DECT) }\end{array}$ \\
\hline positive (CTEPH-positive) & 13 & 0 \\
\hline negative (CTEPH-negative) & 2 & 56 \\
\hline & PPV: 0.867 & NPV: 1.00 \\
\hline
\end{tabular}

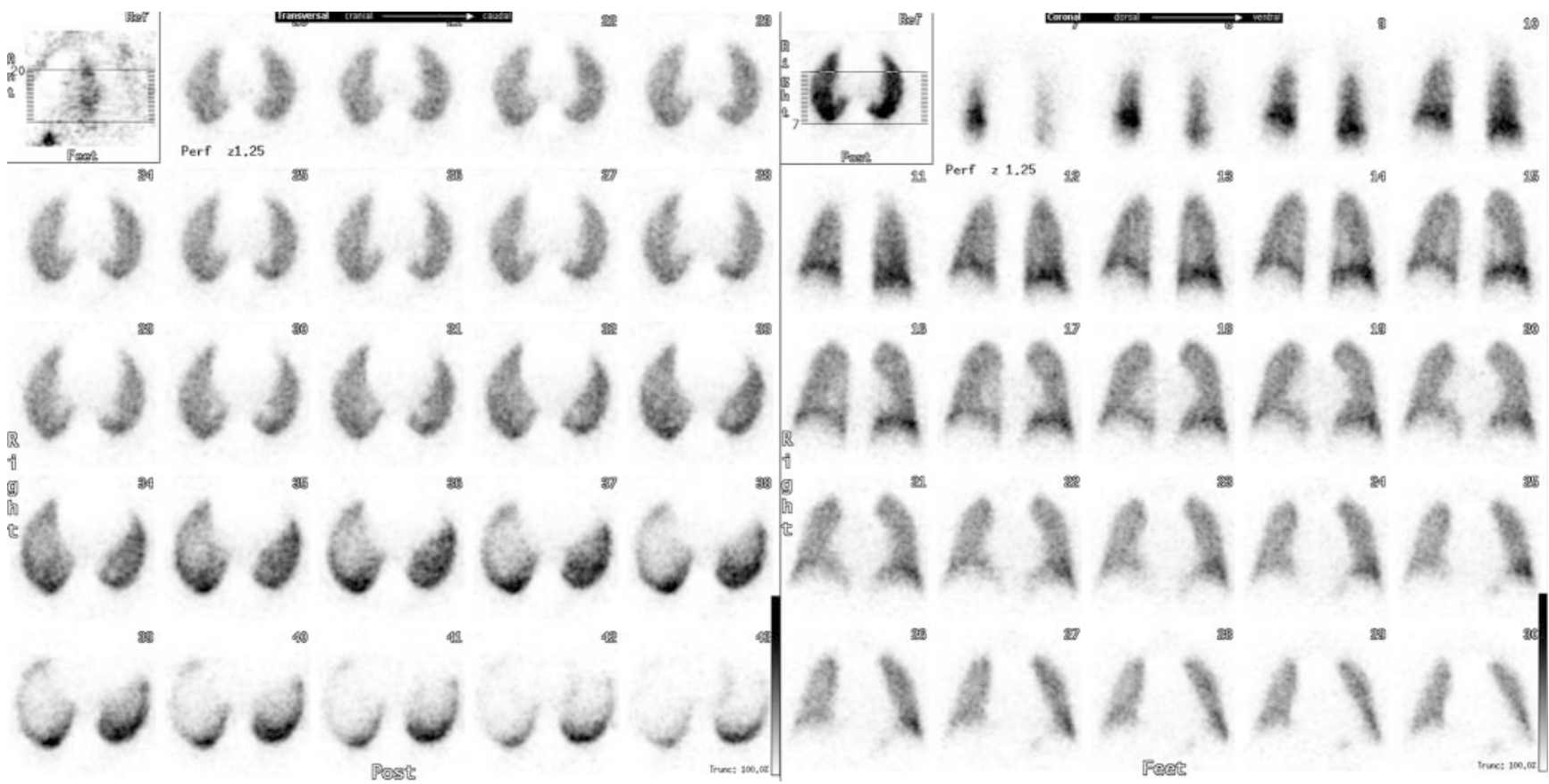

Fig. 5 V/Q-SPECT: Perfusion scintigraphy in axial and coronal planes without evidence of a thrombus or embolism.

positive patients. Based on all of the findings, these patients were ultimately categorized as CTEPH-negative in the CTEPH conference in spite of the presence of small residual thrombi due to their pulmonary artery pressure or lack of perfusion deficits.

\section{Discussion}

There was no significant difference regarding radiation dose between the two examinations in the present study $(1.892 \mathrm{mSv}$ vs. $1.911 \mathrm{mSv}$; bias $=0.01927$ ). This is particularly noteworthy since V/Q-SPECT is currently generally considered to involve less radiation. Even the still relatively new $\mathrm{S} 1$ guidelines regarding lung scintigraphy specify a radiation dose for CTPA that is more than twice as high (2.12 mSv vs. $4.94 \mathrm{mSv}$ ) [11]. Technical advancements are reducing radiation exposure and changing the body of evidence in favor of computed tomography. DECT is not only a method that is equal to the current imaging method of first choice with respect to radiation dose and the visualization of perfusion deficits but also provides numerous additional advantages. The main symptom in CTEPH patients is typically progressive dyspnea at rest. Therefore, lying flat is often very uncomfor- table. Both examinations are performed with the patient in a supine position, but they differ greatly with respect to acquisition time. The time required for a V/Q-SPECT examination is approximately 25-30 minutes [11], while a DECT examination with contrast takes less than 5 minutes. Moreover, the CT examination of the chest simultaneously provides significant additional information. For example, important morphological changes like hypertension of the right ventricle or dilation of the pulmonary trunk as well as any shunt malformations can be detected. Based on cut-off values, the caliber of the pulmonary arteries also provides information about the presence of pulmonary hypertension. Moreover, the lung parenchyma can be evaluated so that important differential diagnoses can be ruled out, such as malignancies, interstitial pulmonary diseases, or inflammatory changes in the parenchyma. At the same time, DECT provides a complete anatomical overview of the thorax and can therefore also rule out restrictive diseases of the lung and pleura. Thus, it must be stated that considerably more information is acquired with an equivalent radiation exposure. A disadvantage of DECT in CTEPH is the phase-dependent loss of iodine map quality. Minor to moderate perfusion deficits are typically compensated by collateral vessels 


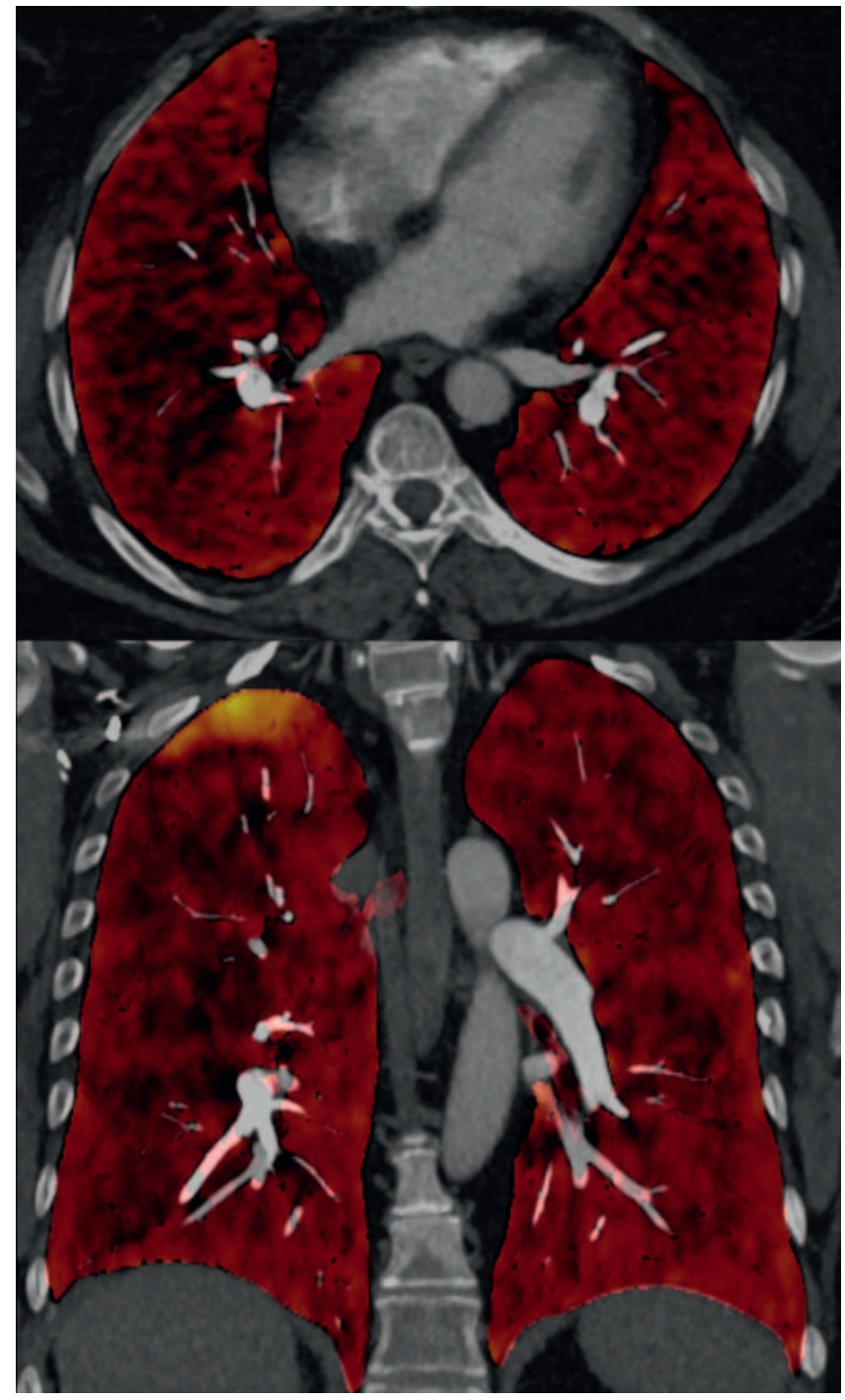

- Fig. 6 lodmap axial and coronal: The knowledge of the present pulmonary arterial web as well as the right-left difference of the iodmap are reasonable grounds for a potential CTEPH.

with a time delay so that images acquired in the late phase can result in false-negative findings based on the iodine map. An examination performed correctly in the pulmonary arterial phase is therefore essential for exact visualization of perfusion including the evaluation of subsegmental defects since CTPA is currently at its limit in this regard.

As already described, many CTEPH patients are correctly diagnosed only in a very late stage of their disease. Treatment and diagnosis are largely also tailored to the late stage. However, it would be desirable to detect CTEPH patients significantly earlier. $\mathrm{V} / \mathrm{Q}$-SPECT, the iodine map of DECT, DSA, and also MRI lung perfusion show only already embolized areas. The following case demonstrates that CTPA can also detect cases that (still) have few symptoms and that would not have been detected using the current diagnostic algorithm based on the guidelines.

- Fig. 5 shows a patient with a completely normal perfusion scintigraphy examination. Based on this, CTEPH would be able to

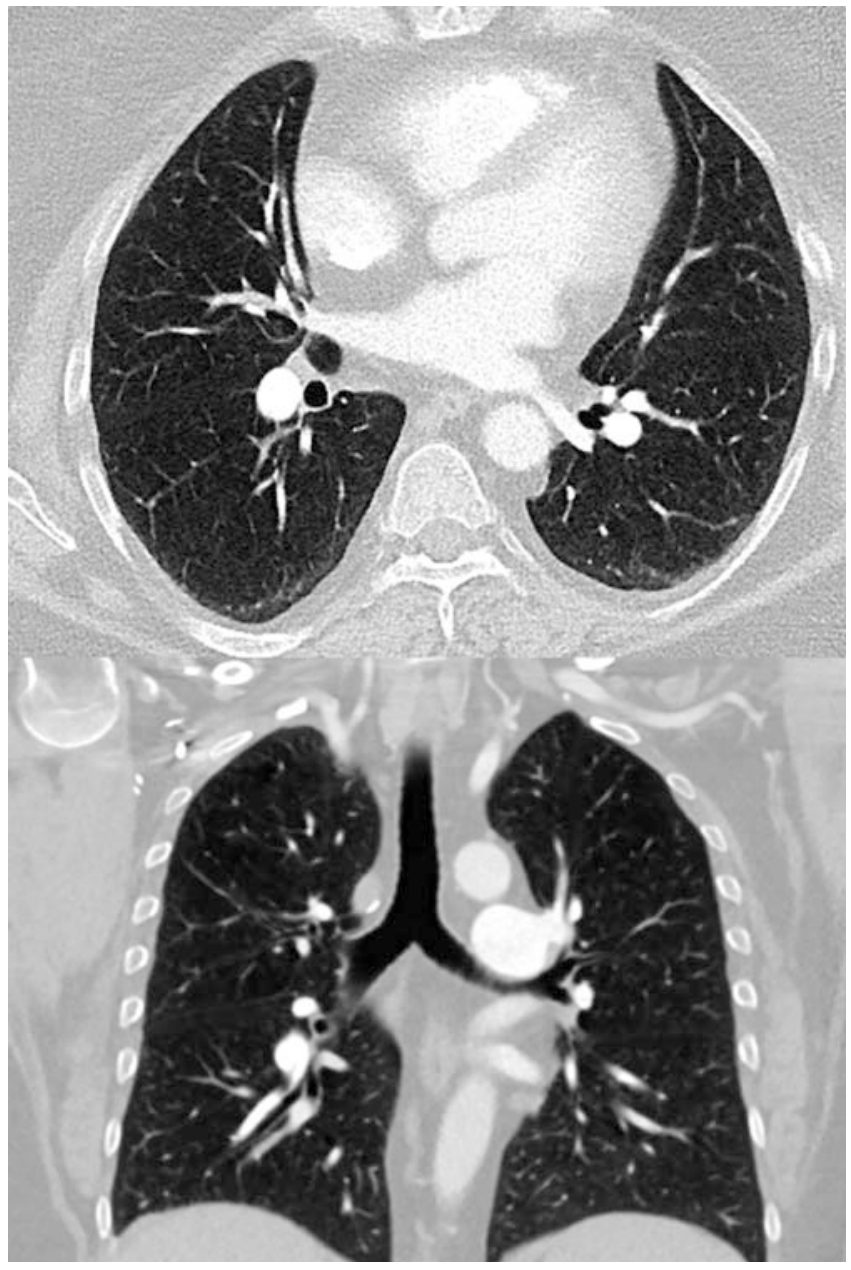

- Fig. 7 Lung window: The lung window shows a slight difference between the right and left lung parenchyma.

be ruled out since a perfusion deficit must be present in at least one segment or in two sub-segments. The corresponding iodine map ( $\triangleright$ Fig. 6) also does not show the classic presentation of CTEPH except for a subtle right-left difference. A slight decrease in density to the disadvantage of the right side is seen in the lung window ( $\triangleright$ Fig. 7). However, a closer look at the CTPA examination ( $\triangleright$ Fig. 8) shows a pulmonary arterial web. Knowing that a thrombus is present, that the patient had a prior acute pulmonary artery embolism, and that an mPAP of $33 \mathrm{mmHg}$ was measured in the right heart catheter, the possibility of CTEPH must be reconsidered. This incidental finding may be decisive for the future management of this patient since he can now be categorized as at risk for $\mathrm{CTEPH}$, can be followed up accordingly, and can receive any required treatment.

Predilection sites for thrombi in pulmonary embolism are not known and could not be deduced from the distribution of perfusion defects in this study. It can be assumed that perfusion deficits follow a statically normal distribution in all lung segments. It was also imaginable that gravity could cause more perfusion deficits in the basal lung segments. However, this assumption was not confirmed in the analysis. On DECT, there was no difference between the upper lobe and the lower lobe of the left lung. The upper lobe 


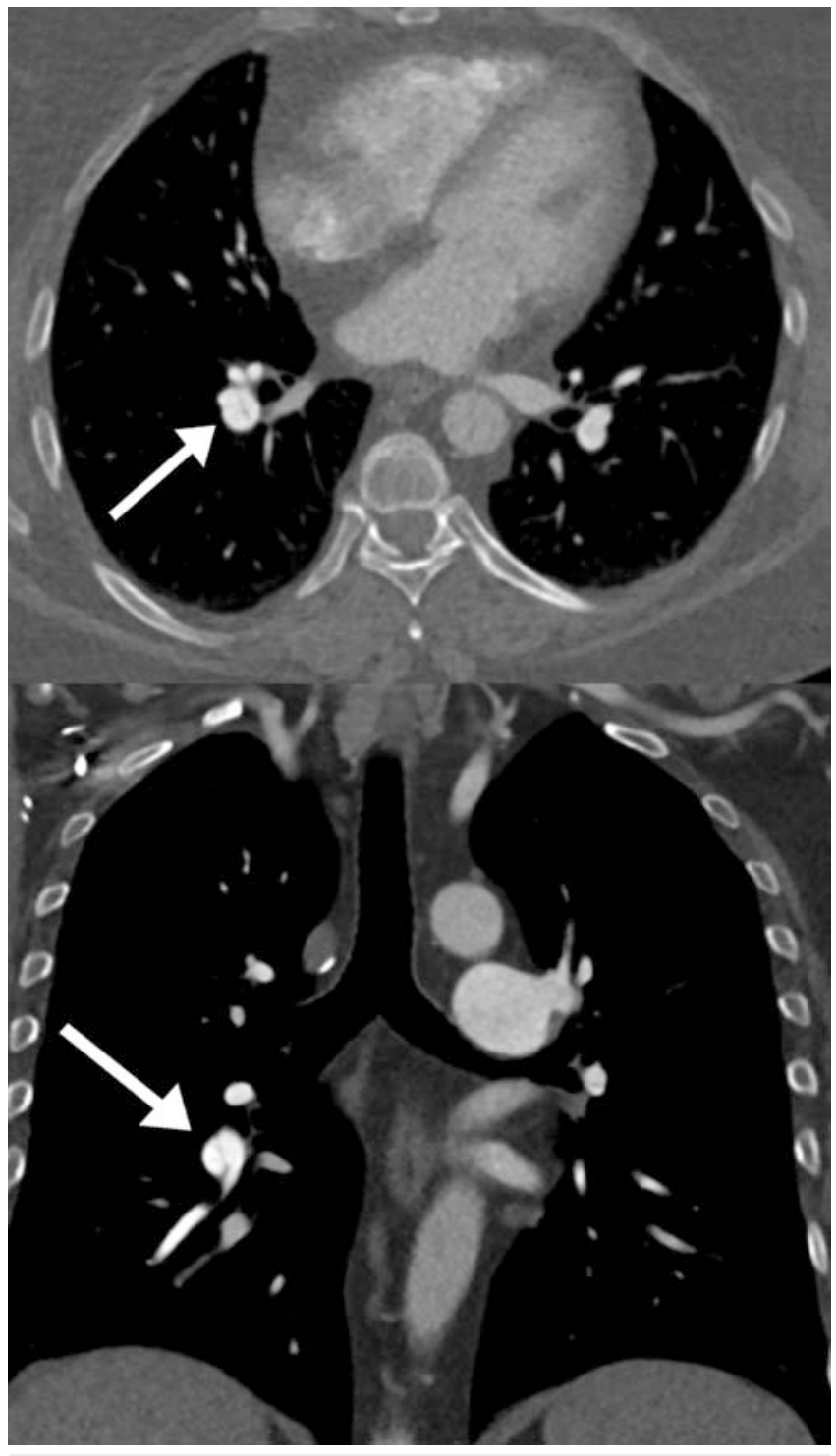

- Fig. 8 CTPA: The detection of the pulmonary arterial web (arrow) gives the decisive information.

was affected even more frequently on V/Q-SPECT. For the right lung, a tendency toward the basal segments is seen on DECT and V/Q-SPECT. However, this result is not statistically significant. The assumption of normal distribution seemed to be the more correct theory. However, segmental evaluation showed that segment four in the right lung was affected significantly more often. On V/Q-SPECT, the result for this segment was even significantly higher than the second standard deviation so that a normal distribution is apparently not present. Multiple causes for the frequent occurrence of embolisms in segment IV are conceivable: Hemodynamic conditions within the lung could be a reason. However, it cannot be ruled out that this segment can be visualized particularly well so that even the smallest findings can be detected.

With an agreement of $92.09 \%$ between DECT and V/Q-SPECT, the result would be able to be rated as "very good". This is limited by a kappa value of 0.5938 , which is similar to the result of Dournes et al., resulting in "moderate" agreement [12]. However, from a mathematical standpoint, it only makes sense to calculate the Cohen's kappa under certain conditions since there is a significant imbalance between embolized and non-embolized segments and the result is consequently incorrectly categorized as primarily incidental and is subsequently corrected with a significant reduction [13]. The results can be better evaluated by analyzing the percentage agreement. The results show a total agreement of $92.09 \%$. However, if only the CTEPH group is taken into consideration, this value is $74.79 \%$. This difference probably cannot be explained solely by the subjectivity of the interpreting physicians but rather indicates that there is a difference between the two modalities. Finally, it must be determined which of the two examination techniques visualizes the extent of CTEPH more precisely. Future studies comparing DECT and V/Q-SPECT with digital subtraction angiography (DSA) as the absolute gold standard could provide clarification here. Ventilation imaging is also technically possible with DECT [9] and would be a further alternative to increase the agreement and/or accuracy of DECT.

On the whole, DECT in combination with the iodine map already has "good" to "very good" agreement compared to the current imaging method of first choice. In addition, all CTEPH patients were detected and DECT had a high sensitivity and specificity so that future implementation in the diagnostic algorithm for CTEPH diagnosis should be taken into consideration. A limitation of this study is the small number of patients.

As described in the current literature, the peak age of CTEPH patients was between 60 and 70 years old. However, the sex distribution of our patients differed from the expected ratio of 1:1. Among the study participants, there was a ratio of approximately 2:1 women to men for the patients with CTEPH as well as for the patients without CTEPH. A p-value of 0.18 indicates a small tendency but the current literature clearly shows that sex is not a risk factor [14-17]. The present result is therefore classified as incidental.

\section{CLINICAL RELEVANCE}

- The higher radiation exposure of computed tomography that is often criticized compared to V/Q-SPECT was able to be refuted and image quality did not suffer in spite of the decrease in radiation dose. Moreover, there was no significant difference regarding radiation exposure between DECT examination and V/Q-SPECT.

- In addition to the suspicion of CTEPH, many additional questions can be answered with a single DECT examination. Therefore, the simultaneous presence of additional cardiopulmonary diseases can be detected.

- A further benefit of DECT for patients is the short examination time (5 minutes) which is a fraction of the time needed in a supine position for V/Q-SPECT.

- For the creation of future diagnostic algorithms, the implementation of a DECT examination with an iodine map before or even instead of V/Q-SPECT should be considered, provided that it is possible to administer a contrast agent containing iodine. Further studies performed at the same time confirm the high agreement between DECT and V/Q-SPECT as well as the excellent sensitivity $[18,19]$. 


\section{Conflict of Interest}

The authors declare that they have no conflict of interest.

\section{References}

[1] Delcroix M, Torbicki A, Gopalan D et al. ERS Statement on Chronic Thromboembolic Pulmonary Hypertension. Eur Respir J 2020. doi:10.1183/13993003.02828-2020

[2] Kim NH, Delcroix M, Jais X et al. Chronic thromboembolic pulmonary hypertension. Eur Respir J 2019; 53: 1801915

[3] Galiè N, Humbert M, Vachiery JL et al. 2015 ESC/ERS Guidelines for the diagnosis and treatment of pulmonary hypertension. Eur Heart J 2016; 37: $67-119$

[4] Olsson KM, Meyer B, Hinrichs J et al. Chronic thromboembolic pulmonary hypertension. Deutsches Arzteblatt international 2014; 111: 856-862

[5] Xu S, Yang J, Zhu Y et al. CTPA, DECT, MRI, V/Q Scan, and SPECT/CT V/Q for the noninvasive diagnosis of chronic thromboembolic pulmonary hypertension. Medicine 2019; 98: e16787

[6] Opitz C, Rosenkranz S, Ghofrani HA et al. ESC-Leitlinie 2015: Diagnostik und Therapie der pulmonalen Hypertonie. Deutsche medizinische Wochenschrift (1946) 2016; 141: 1764-1769

[7] Hoeper MM, Ghofrani HA, Grünig E et al. Pulmonary Hypertension. Deutsches Arzteblatt international 2017; 114: 73-84

[8] Haramati A, Haramati LB. Imaging of Chronic Thromboembolic Disease. Lung 2020; 198: 245-255

[9] Hwang HJ, Hoffman EA, Lee $\mathrm{CH}$ et al. The role of dual-energy computed tomography in the assessment of pulmonary function. European journal of radiology $2017 ; 86: 320-334$
[10] Dournes G, Verdier D, Montaudon M et al. Dual-energy CT perfusion and angiography in chronic thromboembolic pulmonary hypertension: diagnostic accuracy and concordance with radionuclide scintigraphy. European radiology 2014; 24: 42-51

[11] Schümichen C, Schmidt M, Krause T. DGN-Handlungsempfehlung (S1-Leitlinie) Lungenszintigraphie Stand 11/2017 - AWMF-Registernummer: 031-005. 2017

[12] Kwiecien R, Kopp-Schneider A, Blettner M. Concordance analysis: part 16 of a series on evaluation of scientific publications. Deutsches Arzteblatt international 2011; 108: 515-521

[13] Hammann M, Jördens J, Schecker H. Methoden in der naturwissenschaftsdidaktischen Forschung: Übereinstimmung zwischen Beurteilern: Cohens Kappa (к). Springer Verlag. 2014

[14] Yandrapalli S, Tariq S, Kumar J et al. Chronic Thromboembolic Pulmonary Hypertension: Epidemiology, Diagnosis, and Management. Cardiology in review 2018; 26: 62-72

[15] Elwing JM, Vaidya A, Auger WR. Chronic Thromboembolic Pulmonary Hypertension: An Update. Clinics in chest medicine 2018; 39: 605-620

[16] Delcroix M, Kerr K, Fedullo P. Chronic Thromboembolic Pulmonary Hypertension. Epidemiology and Risk Factors. Annals of the American Thoracic Society 2016; 13 (Suppl. 3): S201-S206

[17] Kuznetsov MR, Reshetov IV, Orlov BB et al. Predictors of Chronic Thromboembolic Pulmonary Hypertension. Kardiologiia 2018; 58: 60-65

[18] Kröger JR, Gerhardt F, Dumitrescu D et al. Diagnosis of pulmonary hypertension using spectral-detector CT. International journal of cardiology 2019; 285: 80-85

[19] Masy M, Giordano J, Petyt G et al. Dual-energy CT (DECT) lung perfusion in pulmonary hypertension: concordance rate with $\mathrm{V} / \mathrm{Q}$ scintigraphy in diagnosing chronic thromboembolic pulmonary hypertension (CTEPH). European radiology 2018; 28: 5100-5110 